\title{
A Collaborative Approach to COVID-19 Planning at a Regional Public University
}

\author{
Jeffrey J. Malanson ${ }^{1,2}$ \\ ${ }^{1}$ Department of History, Purdue University Fort Wayne, ${ }^{2}$ Office of Academic Innovation, Purdue University Fort \\ Wayne
}

Cite as: Malanson, J.J. (2022). A Collaborative Approach to COVID-19 Planning at a Regional Public University. Metropolitan Universities, 33(1), 55-63. DOI: 10.18060/25279.

This is an open access article distributed under the terms of the Creative Commons Attribution License.

Editor: Valerie L. Holton, Ph.D.

\begin{abstract}
As Purdue University Fort Wayne (PFW), a regional public university in northeast Indiana, completed the spring 2020 semester fully remote due to COVID-19, university leadership had to determine if there was a path to safely reopening campus and maintaining a low-risk environment for in-person instruction and work for the 2020-21 academic year. To make this determination, PFW engaged in a three-week scenario planning process in which we assembled 22 task forces composed of 140 faculty and staff, approximately $13.5 \%$ of the university's fulltime employees, to identify the challenges and opportunities associated with eight scenarios for how the 2020-21 academic year could play out. Reports and recommendations from the scenario planning process have informed all aspects of the university's COVID-19 planning, implementation, and communication. This paper examines how a highly collaborative planning process, informed by PFW's recently completed strategic planning process, created broad engagement with and awareness of the university's planning efforts, demonstrated the value placed on faculty and staff expertise and input, and helped to build long-term buy-in and trust. This approach also informed PFW's planning processes for the 2021-22 academic year, which emphasized leveraging our COVID-19 experiences to build a better normal for the university.
\end{abstract}

Keywords: scenario planning, COVID-19, strategic planning, regional university, public university 
The COVID-19 pandemic has required institutions of higher education to reevaluate virtually all aspects of their operations and to regularly make critical decisions at what feels like breakneck speeds. One area of innovation for Purdue University Fort Wayne (PFW), a comprehensive metropolitan public university located in northeast Indiana, has been a greater reliance on broadly collaborative planning processes to determine key aspects of university operations. Empowering faculty and staff to establish protocols and develop recommendations for university-wide approaches to challenges, rather than relying on administrative mandates or unitlevel problem solving, has improved the university's response to COVID-19 and fostered greater confidence in and support for key decisions related to pandemic operations. Lessons from the university's pandemic response planning have already shaped our approach to post-pandemic planning and will continue to inform the university's future planning exercises.

As Purdue Fort Wayne neared the end of the spring 2020 semester, the second half of which was fully remote as a necessary response to the early stages of the COVID-19 pandemic, university leadership had to determine if there was a path to safely reopening campus and maintaining a low-risk environment for in-person instruction and work for the 2020-21 academic year. The experience with remote operations in spring 2020 had made clear that our students wanted and needed the opportunity for an in-person educational experience in 2020-21 if that experience could be delivered in a reasonably low-risk manner utilizing the resources the university had at its disposal. Too many students had unreliable access to technology or high-speed internet at home, lacked safe and quiet spaces to complete schoolwork or take online courses, or had too many non-school obligations to be able to effectively manage a fully online course schedule. Our students needed the engagement and support structures that an in-person education, or at least an open campus, afforded.

There were many challenges to answering this planning question. Among the most important beyond the unprecedented nature of the pandemic was that Purdue Fort Wayne had limited administrative capacity to engage in planning on this scale, especially in the short timeframe available before critical decisions for fall 2020 operations needed to be made and communicated to employees, prospective students, current students, parents, and community partners. While part of the Purdue University System, Purdue Fort Wayne is largely autonomous in many aspects of its operations, including its response to COVID-19. There was also the critical question of how to build trust in planning decisions, especially amongst employees, when they were not developed by the full team of medical and infectious disease experts that many larger universities could marshal. 


\section{Scenario Planning and Implementation for 2020-21}

The solution to these challenges was staging a large-scale scenario planning process, the primary goals of which were to determine if there was a path to safely re-opening campus, and identify the salient challenges and opportunities the university would potentially confront in the months ahead. In late April 2020, under the direction of the chancellor, vice chancellor for academic affairs, and an executive steering committee composed of senior administration, the presiding officer of the faculty Senate, and the director of strategic planning as project manager, the university assembled 22 task forces focused on all aspects of university operations. ${ }^{1}$ Each task force was charged with answering a series of planning questions related to the challenges and opportunities associated with eight different scenarios for how the 2020-21 academic year could play out. ${ }^{2}$ A total of 140 faculty and staff members served on the task forces, approximately $13.5 \%$ of full-time employees, with assignments based on areas of functional and/or research expertise while also ensuring that each task force included a broad mix of administrative and academic perspectives. The task forces had a tight timeline of just three weeks in which to complete the planning exercise. This approach to scenario planning was made possible by the university's then-recently completed strategic planning process, which had likewise prioritized broad engagement and participation to build, iterate, and refine a comprehensive university strategic plan in a single calendar year.

This was an intensive process that required a meaningful commitment of time and energy by participating faculty and staff, and it proved to be highly successful both as a planning process and at building trust and awareness. At the beginning of the process, we held an information session for all participants during which we explained the purpose and goals of the process, walked them through specific expectations for their work, and answered questions. We established an intranet site through which we could share documents, resources, and guidance. We built in predetermined check-in times to ensure timely progress and to course correct if needed. Each task force produced a high-quality planning document that enabled university leadership to make key decisions about the pandemic response plan. The broad engagement of

\footnotetext{
${ }^{1}$ Academic Success: fall 2020 Beginners; Academic Success: Graduate Students; Academic Success: Returning Undergraduate Students. Auxiliaries and Food Services; Community Engagement, Governmental Affairs, Development; Diversity, Equity, and Inclusion; DOE, HLC, ICHE, Disciplinary Accreditation; Faculty Scholarship, Promotion and Tenure; fall 2021 Enrollment Funnel; Health, Safety, and Sanitation; Human Resources; Library; Non-credit, Auxiliaries, Resident Organizations; Non-lecture-based Instruction; Student Housing; Student Life and Experience, Athletics; [Purdue] System Relations; Teaching and Learning; Coordination with IU Fort Wayne; Mixed Modality Opportunities and Approaches; Revenue Projections, Expenditure Modifications; ROTC.

${ }^{2}$ The scenarios were informed by our then-limited understanding of how the pandemic was projected to evolve in the coming year and included a fully in-person academic year with no meaningful health and safety modifications, a year of mixed modalities and social distancing, a fully remote year, and scenarios with mixtures of the three and/or major disruptions requiring a transition to fully remote operations.
}

(C) The Author 2022. Published by the Coalition of Urban and Metropolitan Universities. www.cumuonline.org Metropolitan Universities | DOI 10.18060/25279 | February 15, 2022 
our university community helped to create an understanding of the planning effort and demonstrated the value that the university placed on faculty and staff expertise and input. The approach also helped to build long-term trust and buy-in to the planning outcomes, as, even if most people did not have the opportunity or inclination to read the more than 400 pages of planning documents that were created, the process we used was broadly communicated and transparently carried out. At the conclusion of the planning process, we held a virtual town hall open to all members of the university community in which we provided an overview of the planning process, summarized the planning recommendations developed by the task forces, and answered questions.

The recommendations of the scenario planning task forces informed all aspects of the university's subsequent COVID-19 planning and implementation efforts. The full set of planning documents has proven to be a useful reference guide throughout the pandemic, but they were also synthesized to identify core planning themes and priorities that cut across all scenarios and areas of university activity. ${ }^{3}$ The most important theme highlighted in all the planning documents, beyond core health and safety considerations, was communication. The task forces highlighted and emphasized the need to (1) transmit accurate and timely information to all members of our university community through both formal and informal communication channels; (2) ensure that information, guidance, and protocols were clear and consistent in their presentation; and (3) provide the resources necessary for anyone with questions or concerns to find answers and be heard.

The scenario planning process and recommendations had demonstrated the value of having broadly collaborative input into key planning decisions. University leadership determined that it was important to maintain this approach as we iterated the scenario planning recommendations into actionable implementation plans. An early key outcome of the process was the formation of the PFW Prepared Committee. Composed of twenty administrators and faculty and staff members from across the university, the PFW Prepared Committee was charged with using the findings of the scenario planning process to develop recommendations for how to modify the physical infrastructure of the campus and to establish health and safety protocols to achieve and maintain a low-risk environment for in-person instruction and work. While much smaller in scale than the scenario planning process, the central goal of the PFW Prepared Committee remained the same: to leverage faculty and staff expertise and to demonstrate the ongoing weight and influence of employee voices in shaping the university's response to the pandemic.

\footnotetext{
${ }^{3}$ The eight planning themes were communication; accommodations for students, staff, and faculty who cannot or will not return to campus; student and faculty illness; new health, safety, and sanitation procedures; international students; future transition(s) to hybrid/remote operations; applied learning experiences; and how to evaluate, compensate, and reward faculty and staff.
}

(C) The Author 2022. Published by the Coalition of Urban and Metropolitan Universities. www.cumuonline.org Metropolitan Universities | DOI 10.18060/25279 | February 15, 2022 
To address communication, and in accordance with the recommendation made by the Centers for Disease Control and Prevention (CDC), the university appointed a COVID-19 point of contact in June 2020 (2021). The point of contact had two overarching responsibilities: answer questions and respond to concerns from students, staff, faculty, parents, and community stakeholders, and help coordinate the university's overall COVID-19 response. With multiple committees, administrative offices, and academic leaders simultaneously developing and implementing plans and protocols, it was essential to have a single point person to ensure that everyone had access to and acted upon the same information. The sheer volume of information and guidance being compiled and communicated over the summer months also meant that it was easy for people not actively involved in planning and implementation efforts to miss relevant details, so having a direct point of contact accountable for providing answers and ensuring consistency helped to build confidence. One important aspect of this work was virtual PFW Prepared Q\&A sessions, which were held weekly from July 2020 through May 2021 when the university was open, through which important updates could be discussed and questions could be answered. The university also launched a PFW Prepared website and weekly email newsletter dedicated to sharing the latest information about COVID-19 and the university's response.

Between the structured PFW Prepared communication channels and the responsive engagement through the COVID-19 point of contact, the university was thus able to provide the necessary resources to ensure that everyone could understand the what, how, and why of the university's fall 2020 operational plan. Communication would have been a priority even without the scenario planning process, but the consistency of recommendations in that area reinforced the need to be intentional about what was communicated, how it was communicated, and in telegraphing the importance attached to clear and open communication that was responsive to employee and students concerns.

Scenario planning, the PFW Prepared Committee, and other focused planning activities that took place throughout summer 2020 produced a tremendous growth in collaboration between administrative units and academic departments to meet the challenges of the pandemic. Some collaboration was borne out of necessity, such as efforts of the Registrar, Facilities Management, Special Events, the Division of Continuing Studies, and academic departments to completely remake our summer 2020, fall 2020, and spring 2021 course schedules to reflect changes to instructional modality and classroom social distancing expectations. Other collaboration, such as the formation of a new Educational Technologies Team from members of Information Technology Services and the Center for the Enhancement of Learning and Teaching, was driven by a desire to more efficiently meet the needs of students, staff, and faculty. In all cases, it has led to improved communication, the limiting of redundant effort, and suggests improvements in university operations that will outlast the pandemic.

(C) The Author 2022. Published by the Coalition of Urban and Metropolitan Universities. www.cumuonline.org Metropolitan Universities | DOI 10.18060/25279 | February 15, 2022 
Purdue Fort Wayne offered a limited number of courses in face-to-face and hybrid formats during its second summer 2020 session as a pilot for fall 2020. Employees started to return to campus throughout July and August. On Monday, August 24, the university welcomed thousands of students back to campus for the fall semester. While some students, faculty, and staff continued to learn, teach, and work remotely, and most aspects of our operations had been modified to reflect necessary health and safety precautions and protocols, the campus was open. Due to the significant efforts of innumerable people, the campus remained open throughout the 2020-21 academic year.

\section{Proactive Planning for Fall 2021 and Beyond}

Much of the public discourse throughout the first half of 2021 about the positive impact of COVID-19 vaccines was that they would make it possible to go "back to normal." Setting aside the need for broader transformations in higher education necessitated by demographic cliffs, stagnating or declining state financial support, and business model changes, the desirability of a return to "normal" presumes that institutions operated rationally, logically, and efficiently before the pandemic. Even the idea of creating a "new normal" focused primarily on continuing health and safety modifications much more so than embracing meaningful changes to various aspects of how universities behave. Purdue Fort Wayne's planning process for 2021-22 was motivated by how our university community could best leverage the learning and innovation COVID-19 had necessitated to improve the university for the long term. The planning process afforded us the opportunity to intentionally act together to make Purdue Fort Wayne a better place to learn, teach, discover, and work.

The spirit and lessons of the 2020-21 scenario planning process were instructive as the university adapted a smaller scale process in spring 2021 to proactively plan for the 2021-22 academic year. While no one can speak with certainty about the future course of the pandemic, the rollout of highly effective vaccines, combined with the year of experience with modified COVID operations, enabled us to plan around a single scenario for 2021-22 with a reasonable degree of confidence. Factors in this single planning scenario included the COVID-19 vaccine being broadly available to all members of our university community, most students and employees being back on campus, and the re-densification of classrooms and workspaces.

Premised on this baseline planning scenario, the university charged two committees with developing recommendations on how the campus should transition back to more traditional operations: an expanded PFW Prepared Committee and a new task force on academics and student support. The PFW Prepared Committee, which had continued to meet throughout the 2020-21 academic year, added several new members and was renamed the PFW Ready Committee to develop recommendations for fall 2021 operations. The academics and student support task force was created in partnership with the faculty Senate and was composed of 
faculty representatives from each college and key administrators with responsibility for COVID19 response and the instructional enterprise. The leadership of the faculty Senate had been heavily involved in the scenario planning effort in summer 2020 and had served on the PFW Prepared Committee since its formation. The Senate and its educational policy committee played a critical role in evaluating and approving temporary modifications of academic regulations. The formation of the academics and student support task force in spring 2021 was an important additional point of collaboration between the Senate and the administration to develop key recommendations for the future.

The committees established three guiding principles to inform all aspects of the fall 2021 planning process: (1) live the core value established in our strategic plan to put students first by maintaining student-centered expectations for all units; (2) create a better normal for Purdue Fort Wayne; and (3) embrace a wellness mindset (Purdue University Fort Wayne, 2020). Underlying each of these principles was the recognized need to learn from and leverage our COVID-19 experiences to improve the university moving forward.

A great example of this is the omnipresence of Zoom or other virtual meeting and livestreaming software options. The initial instinct of many people has been to either abandon virtual tools to the greatest extent possible once people are back on campus or to continue to live and work almost entirely in the virtual world. In the context of Purdue Fort Wayne, either extreme would constitute a missed opportunity. While we want to be back in person as much as possible, many virtual tools lower barriers to access, facilitate communication and contact, and have received a great deal of positive feedback from students on their efficacy. As a result, use of these tools should be strategically targeted in the future to promote student and employee engagement and support. We must also continue to embrace the culture of experimentation and innovation in our educational enterprise that the pandemic spurred.

\section{Lessons Learned from Collaborative Planning}

While not groundbreaking by any means, one of the core lessons of the scenario planning process has been the importance of giving people meaningful avenues to make their voices heard and then, whenever possible, acting upon what they have told you. The appointment of the PFW Prepared Committee and COVID-19 point of contact were embodiments of this lesson. Throughout the 2020-21 academic year, we carefully considered when, how, and who to ask for feedback on our COVID-19 response efforts that could yield actionable input. We surveyed faculty teaching the first face-to-face courses in summer 2020 to better understand their experience to inform further preparation and communication strategies for fall. During the first month of the fall 2020 semester, we surveyed all face-to-face instructors to ask about their classroom experience to identify what was going well, what challenges they were facing that we could immediately address, and what challenges would require additional planning in the future.

(C) The Author 2022. Published by the Coalition of Urban and Metropolitan Universities. www.cumuonline.org Metropolitan Universities | DOI 10.18060/25279 | February 15, 2022 
The week before the start of the spring 2021 semester, we sent a survey to all employees focused on how we could improve the university's response to COVID-19 moving forward and how they interacted with the various communication channels we had utilized to that point. We took immediate action where able and factored other insights into our 2021-22 planning efforts. For our fall 2021 planning, we sent a survey to both students and faculty asking about how students had experienced the pandemic, what they hoped would change in 2021-22, and what they hoped would stay the same. The results directly shaped both the academics and student support recommendations and the broader framing and messaging attached to them. All surveys were brief and were focused on gathering information that we could directly act upon. Even in situations where we have not been able to implement specific ideas for which people advocated, understanding what they were experiencing directly informed and improved our communication strategies and future planning efforts.

The nature of the COVID-19 crisis forced us to find new ways of working together across institutional silos and continually reminds us of the critical importance of remaining flexible. The scenario planning work to prepare for 2020-21 expedited the process of breaking down barriers, but in the year since we have continued to communicate and collaborate across silos in ways never previously experienced. The recommendations of the scenario planning task forces in the summer of 2020, as well as those of our two planning committees for fall 2021, were an essential foundation for subsequent university decisions and actions, but the course of the pandemic has demonstrated that the best plans are those that are flexible and adaptable to meet rapidly changing conditions. As we prepare for our post-pandemic future, we must continue to plan, act, collaborate, and adapt with an eye toward the whole institution.

\section{Acknowledgments}

While no names are used throughout the article, the planning and implementation processes described in this article were only possible due to the significant efforts of a large portion of the Purdue University Fort Wayne community. The author would specifically like to thank Chancellor Ronald L. Elsenbaumer and Vice Chancellor for Academic Affairs Carl N. Drummond for their encouragement to present information about Purdue Fort Wayne's COVID19 response efforts to CUMU's virtual learning and sharing series and to prepare a version of that presentation for submission as this article. The author would also like to thank the two peer reviewers for their thoughtful reading and suggestions for improvement.

(C) The Author 2022. Published by the Coalition of Urban and Metropolitan Universities. www.cumuonline.org Metropolitan Universities | DOI 10.18060/25279 | February 15, 2022 


\section{References}

Centers for Disease Control and Prevention. (July, 2021). Guidance for Institutions of Higher Education. COVID-19. https:/www.cdc.gov/coronavirus/2019ncov/community/colleges-universities/considerations.html

Purdue University Fort Wayne. (2020). Empowering Transformation: Strategic Plan 2020-25. Strategic Plan and Implementation. https://www.pfw.edu/strategic-plan/documents/PFWCHAN-Strategic-Plan-Trustee-Mtg-Booklet-508-DIGITALv2.pdf 